\title{
Beneficial and Limiting Factors Affecting Return to Work After Total Knee and Hip Arthroplasty: A Systematic Review
}

\author{
P. P. F. M. Kuijer - M. J. P. M. de Beer • \\ J. H. P. Houdijk · M. H. W. Frings-Dresen
}

Published online: 20 August 2009

(c) The Author(s) 2009. This article is published with open access at Springerlink.com

\begin{abstract}
Introduction A large number of patients undergoing total knee (TKA) and hip (THA) arthroplasties are of working age at the time these procedures are performed. The objective of this study was to systematically review literature on the beneficial and limiting factors affecting return to work in patients undergoing TKA or THA. Method Pubmed and Embase were systematically searched to find studies that described factors that influence return to work (RTW) after surgery. The following inclusion criteria had to be met: (1) inclusion of patients with primary or revision TKA or THA; (2) description of return to work after surgery or employment status; and (3) description of a beneficial or restricting factor affecting return to work. Results Only three studies were found that fulfilled the three inclusion criteria. Three factors were discussed: (1) the mini-posterior approach compared to the two-incision approach; (2) patient movement restrictions after surgery compared to no restrictions; and (3) patient discharge based on guidelines compared to discharge without guidelines. Conclusions This systematic review revealed that knowledge is sparse regarding beneficial or limiting factors affecting return to work after TKA or THA. Despite that, the results suggests that the two-incision approach is beneficial, patient movement restrictions are limiting, and patient discharge guidelines have no effect on the time patients take to RTW.
\end{abstract}

P. P. F. M. Kuijer $(\bowtie) \cdot$ M. H. W. Frings-Dresen

Coronel Institute of Occupational Health, People and Work

Outpatient Clinic, Academic Medical Center,

University of Amsterdam, P.O. Box 22700, 1100 DE

Amsterdam, The Netherlands

e-mail: p.p.kuijer@amc.nl

M. J. P. M. de Beer · J. H. P. Houdijk

Research Institute MOVE, Faculty of Human Movement

Sciences, VU University, Amsterdam, The Netherlands
Keywords Arthroplasty · Knee · Hip · Work · Employment · Intervention

\section{Introduction}

Although joint replacements, such as total knee (TKA) and hip (THA) arthroplasties, are most common among older people, a Finnish study reported that $45 \%$ of prosthetic implant surgeries are performed on people under the age of 65 [1]. Two older studies reported that two-thirds of patients with hip prostheses try to remain at their job or return to it, because working represents a vital dimension of their quality of life $[2,3]$. While these figures might be a bit out of date and might not be representative of all countries, they do demonstrate that there are likely a fair number of people in the working population with joint implants. Moreover, these numbers will likely continue to increase as many people are now working later into life, the Western population is getting older, and rising obesity rates are increasing the risk of one of the primary reasons for joint replacement, i.e., osteoarthritis [4, 5].

Research has also shown that the ability to work represents an important aspect of people's lives; while working, people feel more productive and feel a sense of structure in their lives [6]. Moreover, returning to work after surgery improves patients' economic situation and also reduces the economic burden on society. Considering the large and likely increasing population undergoing TKA or THA during the working years, and the beneficial effects associated with returning to work, it is important to understand how best to help these patients return to work after surgery.

Most studies in the field of joint replacements, however, are primarily interested in variables closely linked to the 
functioning of the joint, like range of motion or muscle power in the affected limb [7-9]. On a functional level, other variables have been studied, like duration of hospital stay, performance of daily activities, pain scores, self perceived functional outcome, time to walk, and patient satisfaction $[10,11]$. Some studies have even looked into the effect of TKA and THA on working status, specifically [12, 13], or have used 'return to work' as a dependent variable $[14,15]$; however, there are no articles summarizing these latter results. Thus, it remains unclear what effect TKA or THA has on working status in general, or whether there are specific factors, like pre-operation sick leave, length of hospital stay, specific rehabilitation programs, or patient intrinsic factors, that accelerate or hamper return to work after TKA or THA. Hence, the objective of this study was to systematically review recent literature on the beneficial and limiting factors affecting return to work in patients undergoing TKA or THA.

\section{Methods}

\section{Search Strategy}

A systematic search was performed in two bibliographic databases (Pubmed and Embase). The search was restricted to studies done in the 10 years prior to the year the search was performed (March 1998-2008), as it seemed questionable whether older studies would still be relevant. Furthermore, studies had to be written in English, French, German or Dutch, had to have an abstract, and had to be performed in humans.

The search terms used in Pubmed are described in Table 1. For effective searching, medical subject headings [MeSH] were used. Furthermore, the MeSH themselves were also used as free text words in the search and were supplemented with other free text words. The list of text words was composed with the use of a thesaurus, the synonyms used by the MeSH database, and the abstracts of several articles, found in a pilot search, that were related to the topic. The three columns were combined using the Boolean command OR. The terms used in Embase are also listed in Table 1. In Embase, the EMTREE system-a system comparable to the MeSH system, but with slightly different terms-was used for effective searching. The EMTREE words were also used in searches as free text words and, as in Pubmed, they were supplemented with other free text words. As in the Pubmed search, the columns were combined using the Boolean command OR.

The references of the articles found were checked for other possibly useful articles that were not found with the original search strategy.

\section{Inclusion Criteria}

The following inclusion criteria had to be met for studies to be included in this systematic review:

1. Inclusion of patients with primary or revision TKA or THA.

2. Description of return to work after surgery or employment status.

3. Description of a beneficial or restricting factor affecting return to work.

TKA was defined as replacement of both the femoral and acetabular part of the knee joint. THA was defined as replacement of both the cup and the stem part of the hip joint.

\section{Selection}

The assessment of whether studies met the inclusion criteria was based on study titles and abstracts. The full text of articles seeming to meet the criteria was then read to check their value for this systematic review. When necessary, the author (M. B.) consulted the other authors (P. K. and M. F. D.) about certain articles, and a consensus had to be reached between all three authors before an article was used for the review.

\section{Data Extraction}

The information extracted from articles that met the inclusion criteria included authors' names, year of publication, country of origin of the study cohort, number of subjects in the study, age of the subjects, number of patients working prior to surgery, type of operation (primary or revision), technique used, components used, number of patients working postoperatively, beneficial or limiting factor(s), observation time, and effects of factors on return to work. This information is summarized in Table 2.

\section{Results}

In Pubmed, 189 articles were found, of which 20 were reviews. After analysing the abstracts, 27 articles seemed to match the inclusion criteria for this study. Of these 27 articles, eight were related to TKA, 17 were related to THA, and two were related to both TKA and THA. In Embase, 457 articles were found, of which only 33 were deemed relevant. Of these 33 articles, 8 were about TKA, 21 were about THA, and 4 were about both TKA and THA. Of the 60 articles found in both database searches, nine were duplicates. After applying the inclusion criteria, only 25 articles examined working status, and only three of 
Table 1 Search terms used in Pubmed and Embase for total hip arthroplasty, total knee arthroplasty and work
Pubmed: $[$ Mesh $]=$ medical subject headings; $*=$ free symbol; AND = boolean command; Embase:

" = EMTREE word; AND $=$ boolean command

\begin{tabular}{lll}
\hline THA & TKA & Work \\
\hline $\begin{array}{l}\text { Pubmed } \\
\text { “Arthroplasty, replacement, hip" } \\
\text { [mesh] }\end{array}$ & $\begin{array}{l}\text { "Arthroplasty, replacement, } \\
\text { knee"[mesh] } \\
\text { Prosthesis AND hip }\end{array}$ & "Work"[mesh] \\
$\begin{array}{l}\text { Arthroplasty AND hip } \\
\text { Replacement AND hip }\end{array}$ & $\begin{array}{l}\text { Arthroplasty AND knee } \\
\text { Replacement AND knee }\end{array}$ & "Employment”[mesh] \\
& & "Occupations"[mesh] \\
& & Worka* \\
& & Worke* \\
& & Work'* \\
& & Workg* \\
& & Worki* \\
& & Workp* \\
'Arthroplasty' AND & 'Arthroplasty' AND & Occupation* \\
Replacement AND 'hip' & Replacement AND 'knee' & Job \\
'Prosthesis' AND 'hip' & 'Prosthesis' AND 'knee' & Profession \\
'Arthroplasty' AND 'hip' & 'Arthroplasty' AND 'knee' & 'Work' \\
'Replacement' AND 'hip' & & 'Employment' \\
\hline
\end{tabular}

these 25 actually investigated a beneficial or restricting factor regarding return to work in patients undergoing TKA or THA. Inspection of the references of these three articles did not reveal any additional relevant articles.

All three articles examined factors affecting employment status after THA; one of these articles also looked at the effect of such factors on return to work after TKA. Table 2 shows a summary of the study characteristics and of the results of the three studies.

Of the three articles found, the first examined the effect of operating technique-the two-incision approach versus the mini-posterior approach-on the dependent variable 'return to work'. Tanavalee et al. [16] compared a group of 35 patients in which the two-incision approach was used to a group of 35 patients in which the mini-posterior approach was employed. Both approaches belong to the category of minimally invasive operating techniques; however, they differ in the number of surgical incisions used. The miniposterior approach requires only one incision, while the two-incision approach obviously requires two. All THAs were performed by the same surgeon, and the same single cementless hip system was used for every patient (Trilogy acetabular component and Versys Fiber Metal Taper femoral component, Zimmer, Warsaw, IN, USA). The protocol for drug administration, including operative anesthetics, was also the same across patients. The groups were not randomized nor matched, yet they were tested for differences in age, sex, the number of hips operated, equal distribution of sides, and body mass index (BMI). Only sex was found to be significantly different between the two groups, with 20 males and 15 females in the mini-posterior group and 8 males and 27 females in the two-incision group. Tanavalee et al. [16] found that the two-incision group returned to work sooner after surgery than the miniposterior group at a mean [standard deviation (SD)] of 3 (1.3) weeks, compared to a mean (SD) of 7 (2.1) weeks for the mini-posterior approach $(P<0.01)$. These results suggested that the two-incision approach represents a beneficial factor with respect to returning to work, as compared to the mini-posterior approach.

The second article, by Peak et al. [17], described the effect of patient restrictions after surgery on functional recovery, including return to work. Peak et al. [17] randomly assigned 265 patients with a total of 303 THAs to a restricted group ( $n=152$ THAs) or an unrestricted group $(n=151$ THAs). All patient were expected to limit the range of motion in the hip for the first 6 weeks to $<90^{\circ}$ of flexion and $45^{\circ}$ of external and internal rotation, and to avoid adduction. All patients were allowed to bear weight as tolerated and were allowed to use walking aids when needed. Management of the patients in the restricted group also included: the placement of an abduction pillow in the operating room before bed transfers; the use of pillows to maintain abduction while in bed; and the use of elevated toilet seats and elevated chairs in the hospital, rehabilitation facility, and at home. Additionally, patients were prevented from sleeping on their side, driving, or being driven in an automobile. Patients in the unrestricted group 


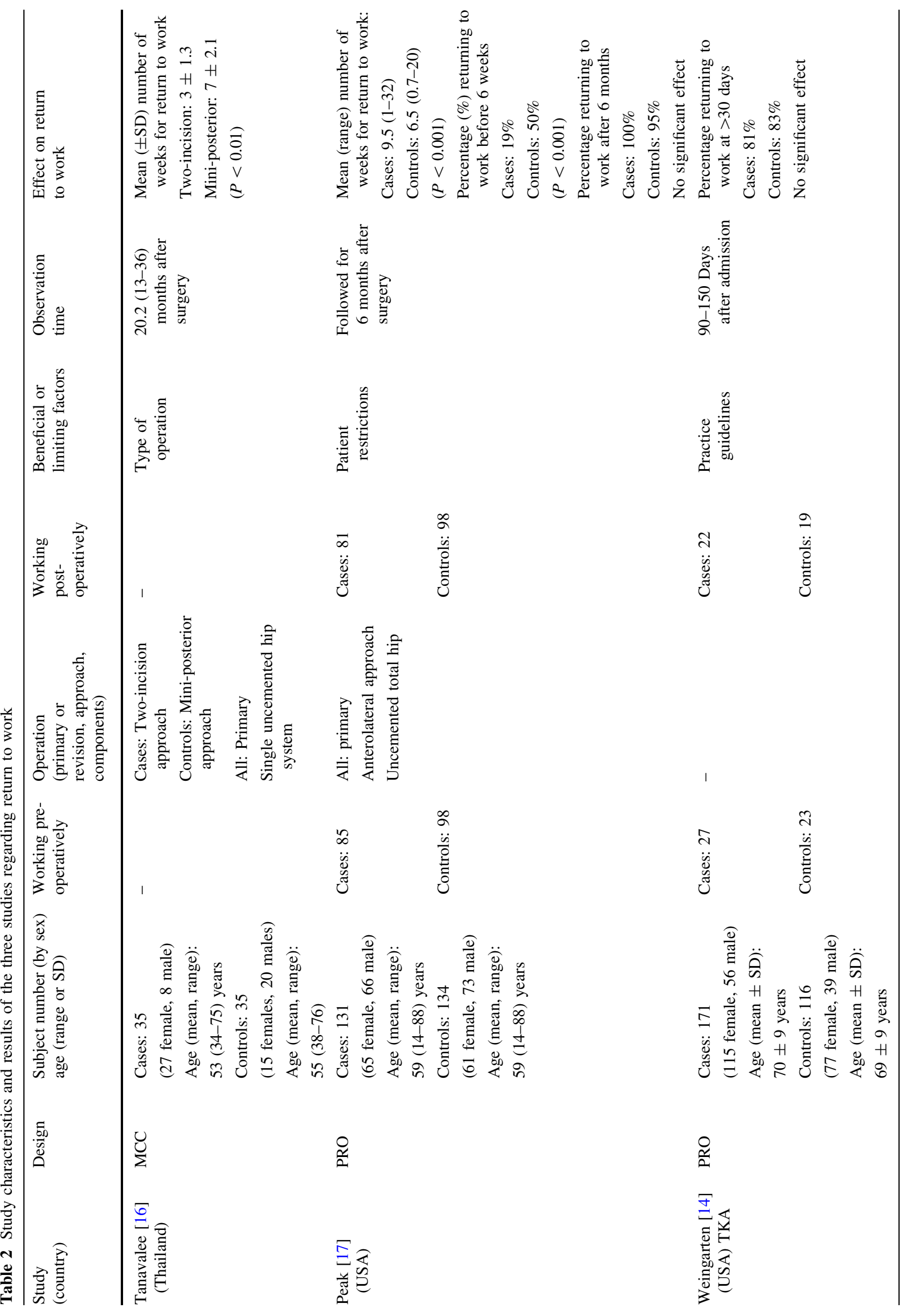




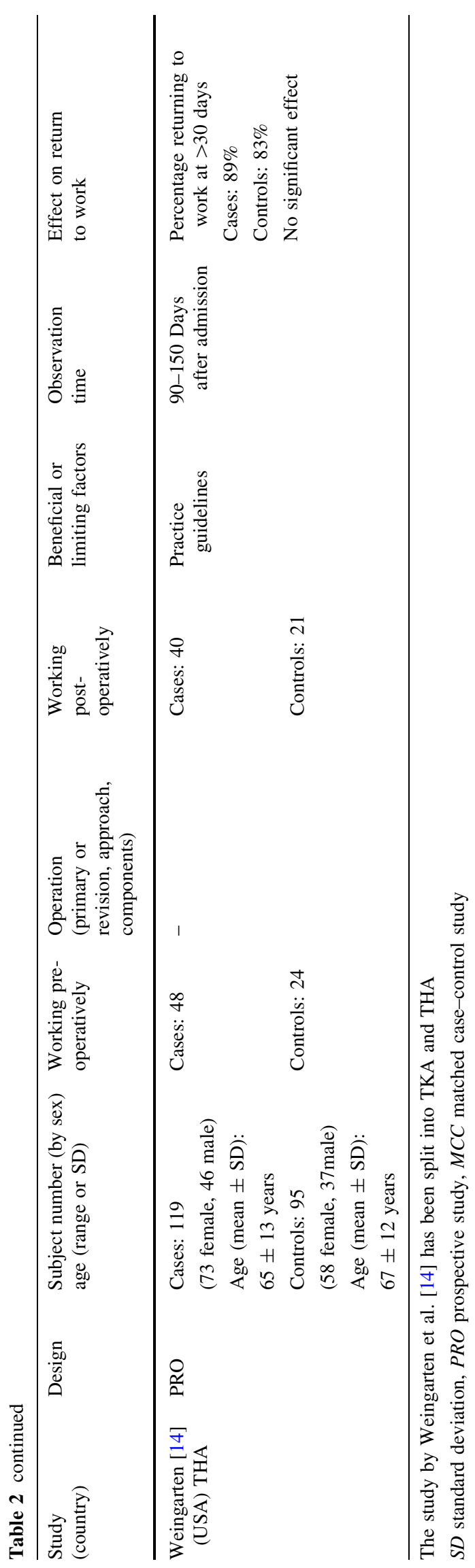

were free to use any of the additional equipment if they desired.

Peak et al. [17] found that the unrestricted patients returned to work after 6.5 weeks, with a range of 0.7-20.0 weeks, while the restricted group returned to work after 9.5 weeks, with a range of 1.0-32.0 weeks $(P<0.001)$. They also found that $50 \%$ of the patients in the unrestricted group and $18 \%$ in the restricted group returned to work within 6 weeks after surgery $(P<0.001)$. Moreover, all 98 of the working patients in the unrestricted group had returned to their usual jobs after 6 months of follow-up, compared to 81 out of 85 patients in the restricted group $(P=0.096)$. Their findings suggested that restricting patients with this specific protocol prolonged the time required to return to work. In other words, restricting patients according to this protocol was a limiting factor regarding return to work after THA.

The third article, by Weingarten et al. [14], investigated the effect of discharging patients characterized as "low risk" (based on practice guidelines for length of hospital stay) on the functional recovery of patients after both TKA and THA. It is quite possible that earlier discharge from the hospital might affect recovery positively, by forcing patients to become more active at an earlier time. However, it is also quite possible that earlier discharge affects recovery negatively, due to insufficient rest and medical attention. The guidelines for discharge with THA [18] and TKA [19] were described and investigated retrospectively in two earlier studies, which showed that length of hospital stay decreased with the use of these guidelines. According to the guidelines, if patients are qualified as "low risk," they are to be discharged on post-operative day 5 or 4 for TKA and THA, respectively. Weingarten et al. [14] studied 560 patients with three different diagnoses, from six different hospitals. Their cohort included 214 patients undergoing THA, 59 with hip fractures, and 287 undergoing TKA. The hip fracture group will not be mentioned again. The patients were divided between a baseline group and an intervention group, based on their date of admission to the hospital. For the THA group, 95 patients were placed in the baseline group and 119 patients in the intervention group. For the TKA group, 116 and 171 patients were in the baseline and intervention groups, respectively.

The study looked at the effect of introducing guidelines for identifying "low risk" patients that might be suited for early discharge. The guidelines were explained to the physicians and nursing staff prior to the baseline period of 3 months, followed by the intervention period of 6 months. Weingarten et al. [14] examined whether there were differences between the baseline and intervention groups regarding age, gender, and employment, and found none, with the exception of employment; more patients in the total hip replacement intervention group were employed -40 
patients versus 25 in the baseline group $(P=0.03)$. They did not find any difference in the percentage of patients that returned to work within 30 days after admission; 89 and $83 \%$ returned to work in the THA baseline and THA intervention groups, respectively $(P=0.70)$, while 81 and $83 \%$ returned to work in the TKA baseline and TKA intervention groups, respectively $(P>0.99)$. These findings suggested that discharging patients in the "low risk" group based on practice guidelines is not a beneficial or limiting factor regarding return to work after TKA or THA.

\section{Discussion}

This systematic review revealed that knowledge is sparse regarding beneficial or limiting factors affecting return to work after TKA or THA. Although the search strategy seems to be very sensitive, only three articles were identified that investigated factors influencing return to work after TKA or THA. Results showed that the two-incision approach might be favored when a timely return to work is desired, and that restricting a patient in his or her movements after surgery may be a limiting factor regarding return to work after THA. Moreover, discharging patients based on practice guidelines had no influence on return to work after either TKA or THA.

The most remarkable finding of this systematic review, however, was the almost complete lack of literature on beneficial or limiting factors affecting return to work after TKA and THA. There were significantly more studies addressing the relationship between joint replacements and return to work, but even those were sparse compared to the comprehensive literature on joint replacements in general. We can think of two explanations for this lack of attention.

First, many studies reported high rates of patients returning to work, which could imply that there is little need to help patients get back to work. For example, Mobasheri et al. [13] and Berger et al. [20], examining THAs, and Jahromi et al. [21], examining TKAs, found that almost all patients returned to work, and when patients did not, it was for reasons unrelated to the joint replacement. There are, however, also studies reporting return to work rates of slightly over 50\%, e.g., Espehaug et al. [22] for THA and Jorn et al. [12] for TKA. Similar to the percentage of patients reported to return to work, the time elapsed prior to returning to work varies significantly between studies as well. Various studies have reported times ranging from a little over a week [22], to little more than a month [15], to several months [13]. However, it would seem that, even if the majority of patients return to work swiftly, these statistics might still be improved by understanding which factors contribute to an optimal return to work.
A second reason for the lack of information regarding return to work could be the indirect assumption that people return to work when their hip or knee is functioning properly again. As mentioned in the introduction, most studies focus on functional recovery of the joint [7-9] and/ or on subjective reports $[10,11]$. It is quite normal to assume that, if joint function improves, people can resume their normal lives, including their work. However, this may not always be the case. It could be that, despite nearly normal range of motion, working activities remain difficult to perform for a certain period of time. Given this, functional joint recovery should not be assumed a priori to be equivalent to returning to work. Therefore, return to work should be a topic of interest in clinical care.

The question remains, however, what exactly should be investigated. According to the International Classification of Functioning model (ICF) proposed by the World Health Organization (WHO), working-ability is based on six, mutually related, components: disease and disorder; functions and structures; activities pertaining to the execution of a task or action by an individual; participation pertaining to the involvement in a life situation; environmental factors; and personal factors. These components all contain different aspects that influence participation in work [23].

This review demonstrated that only three factors related to treatment of joint disease have been investigated with regard to the patient's return to work: type of operation, patient restrictions, and discharge based on guidelines. Based on the literature on TKA and THA, the following factors have been suggested to be important in optimizing return to work after TKA or THA: comorbidity [24, 25], previous TKA or THA, or either revision [22], type of component used [26-28], kind of disease [29, 30], and possible treatment after surgery [20]. It goes without saying that the factors already identified require extra research as well. Several articles $[15,20,31]$ in addition to that by Tanavalee et al. [16] indicate that the type of operation could be very important. These are all primary factors related to disease, according to the ICF model. Under this model, other aspects, such as the type of work performed (environment), should also be taken into account, especially when the work environment involves exposure to risk factors for knee and hip osteoarthritis, such as kneeling or lifting [32, 33].

Open Access This article is distributed under the terms of the Creative Commons Attribution Noncommercial License which permits any noncommercial use, distribution, and reproduction in any medium, provided the original author(s) and source are credited.

\section{References}

1. Paavolainen P, Hamalainen M, Mustonen H, Slatis P. Registration of arthroplasties in Finland. A nationwide prospective project. Acta Orthop Scand Suppl. 1991;241:27-30. 
2. Gerson EM. Quality of life. Am Sociol Rev. 1976;41:793-806.

3. Najman J, Levine S. Evaluating the impact of medical care and technology on the quality of life: a review and critique. Soc Sci Med. 1982;15:107-15.

4. Taubes G. Demographics: as obesity rates rise, experts struggle to explain why. Science. 1998;29:1367-8.

5. March LM, Bagga H. Epidemiology of osteoarthritis in Australia. Med J Aust. 2004;1(5 Suppl):6-10.

6. O'Brien GE, Feather NT. The relative effects of unemployment and quality of employment on the affect, work values and personal control of adolescents. J Occup Psychol. 1990;63:151-65.

7. Bryne JM, Gage WH, Prentice SD. Bilateral lower limb strategies used during a step-up task in individuals who have undergone unilateral total knee arthroplasty. Clin Biomech. 2002;17:580-5.

8. Hesse S, Werner C, Seibel H, von Frankenberg S, Kappel EM, Kirker $S$, et al. Treadmill training with partial body-weight support after total hip arthroplasty: a randomized controlled trial. Arch Phys Med Rehabil. 2003;84:1767-73.

9. Öberg T, Öberg U, Sviden G, Nordwall Persson A. Functional capacity after hip arthroplasty: a comparison between evaluation with three standard instruments and a personal interview. Scand J Occup Ther. 2005;12:18-28.

10. Moran M, Khan A, Sochart DH, Andrew G. Evaluation of patient concerns before total knee and hip arthroplasty. J Arthroplasty. 2003; 18:442-5.

11. Ebinesan AD, Sarai BS, Walley G, Bridgman S, Maffulli N. Total knee arthroplasty: good agreement of clinical severity scores between patients and consultants. BMC Musculoskelet Disord. 2006;7:61.

12. Jorn LP, Johnsson R, Toksvig-Larsen S. Patient satisfaction, function and return to work after knee arthroplasty. Acta Orthop Scand. 1999;70:343-7.

13. Mobasheri R, Gidwani S, Rosson JW. The effect of total hip replacement on the employment status of patients under the age of 60. Ann R Coll Surg Engl. 2006;88:131-3.

14. Weingarten S, Reidinger MS, Sandhu M, Bowers C, Ellrodt AG, Nunn C, et al. Can practice guidelines safely reduces hospital length of stay? Result from a multicenter interventional study. Am J Med. 1998;105:33-40.

15. Pagnano MW, Trousedale RT, Meneghini RM, Hanssen AD. Patient preferred a mini-posterior THA to a contralateral twoincision THA. Clin Orthop Relat Res. 2006;453:156-9.

16. Tanavalee A, Jaruwannapong $S$, Yuktanandana $P$, Itiravivong $P$. Early outcomes following minimally invasive total hip arthroplasty using a two-incision approach versus a mini posterior approach. Hip Int. 2006;16(Suppl 4):17-22.

17. Peak EL, Parvizi J, Ciminiello M, Purtill JJ, Sharkey PF, Hozack $\mathrm{WJ}$, et al. The role of patient restrictions in reducing the prevalence of early dislocation following total hip arthroplasty. A randomized prospective study. J Bone Joint Surg Am. 2005;87: 247-53.
18. Weingarten SR, Riedinger M, Connor L, Siebens H, Varis G, Alter A, et al. Hip replacement and hip hemiarthroplasty surgery: potential opportunities to shorten lengths of hospital stay. Am J Med. 1994;97:208-13.

19. Weingarten SR, Conner L, Riedinger M, Alter A, Brien W, Ellroft AG. Total knee replacement: a guideline to reduce postoperative length of stay. West J Med. 1995;163:26-30.

20. Berger RA, Jacobs JJ, Meneghini RM, Della Valle C, Paprosky W, Rosenberg AG. Rapid rehabilitation and recovery with minimally invasive total hip arthroplasty. Clin Orthop Relat Res. 2004;429:239-47.

21. Jahromi I, Walton NP, Dobson PJ, Lewis PL, Campbell DG. Patient-perceived outcome measures following unicompartmental knee arthroplasty with mini-incision. Int Orthop. 2004;28:286-9.

22. Espehaug B, Havelin LI, Engesaeter LB, Langeland N, Vollset SE. Patient satisfaction and function after primary and revision total hip replacement. Clin Orthop Relat Res. 1998;351:135-48.

23. Slebus FG, Sluiter JK, Kuijer PPFM, Willems JHBM, FringsDresen MHW. Work-ability evaluation: a piece of cake or a hard nut to crack? Disabil Rehabil. 2007;29:1295-300.

24. Toomey HE, Toomey SD. Hip arthroplasty in chronic dialysis patients. J Arthroplasty. 1998;13:647-52.

25. Nakai T, Masuhara K, Matsui M, Ohzono K, Ochi T. Therapeutic effect of transtrochanteric rotational osteotomy and hip arthroplasty on quility of life of patient with osteonecrosis. Arch Orthop Trauma Surg. 2000;120:252-4.

26. Langdon IJ, Bannister GC. Cemented hip replacements in patients younger than 50 years: 16-24 year results. Hip Int. 1999; 9:151-3.

27. Rodrigo JJ, Hazelwood SJ, Farver TB, Nyman JS. Total knee replacement with interlocking stems. Clin Orthop Relat Res. 2001;392:139-46.

28. Yoo JJ, Kim Y, Yoon KS, Koo K, Kim JW, Nam KW, et al. Contemporary alumina-on-alumina total hip arthroplasty performed in patients younger than forty years: a 5-year minimum follow-up study. J Biomed Mater Res Part B Appl Biomater. 2006;78:70-5.

29. Sarkar MR, Billhanz E, Wachter N, Kinzl L, Bischoff M. Longterm outcome of secondary joint replacement after acetabular fracture. Eur J Trauma. 2001;27:301-8.

30. Sarkar MR, Wachter N, Kinzl L, Bischoff M. Acute total hip replacement for displaced acetabular fractures in older patients. Eur J Trauma. 2004;30:296-304.

31. Procyk S. Initial results with a mini-posterior appraoch for total hip arthroplasty. Int Orthop. 2007;31(Suppl 1):17-20.

32. Bierma-Zeinstra SMA, Koes BW. Risk factors and prognostic factors of hip and knee osteoarthritis. Nat Clin Pract Rheumatol. 2007;3:78-85.

33. Jensen LK. Hip osteoarthritis: influence of work with heavy lifting, climbing stairs or ladders, or combining kneeling/squatting with heavy lifting. Occup Environ Med. 2008;65:6-19. 